\title{
Quenched free energy in random matrix model
}

\author{
Kazumi Okuyama \\ Department of Physics, Shinshu University, \\ 3-1-1 Asahi, Matsumoto 390-8621, Japan \\ E-mail: kazumi@azusa.shinshu-u.ac.jp
}

ABSTRACT: We compute the quenched free energy in the Gaussian random matrix model by directly evaluating the matrix integral without using the replica trick. We find that the quenched free energy is a monotonic function of the temperature and the entropy approaches $\log N$ at high temperature and vanishes at zero temperature.

KeYwords: Matrix Models, Random Systems

ARXiv EPrint: 2009.02840 


\section{Contents}

1 Introduction 1

2 Quenched free energy in Gaussian matrix model 3

2.1 High temperature regime 3

2.2 Low temperature regime 4

3 Numerics for $N=2,3 \quad 5$

4 Comment on the replica method $\quad 7$

$\begin{array}{llr}5 & \text { Discussion } & 9\end{array}$

\section{Introduction}

Recently, it is shown that the path integral of Jackiw-Teitelboim (JT) gravity [1, 2] is equivalent to a certain double-scaled matrix model [3]. From the viewpoint of holography, this implies that the holographic dual of JT gravity is not a single quantum mechanical system but an ensemble of systems with random Hamiltonians. Moreover, it is realized in [4] that the Euclidean wormhole connecting different boundaries of spacetime plays an important role in explaining the so-called "ramp" of the spectral form factor $[5,6]$ in the Sachdev-Ye-Kitaev (SYK) model [7, 8]. The importance of the wormhole in quantum gravity is also emphasized in the recent computation of the Page cure using the replica method $[9,10]$, where the inclusion of the so-called replica wormhole is essential for the resolution of the apparent paradox in the original Hawking's calculation. ${ }^{1}$

In recent papers $[11,12]$ the replica method is applied to the computation of the free energy in JT gravity. As emphasized in [11], this problem is very interesting to reveal the role of replica wormholes and explore the possibility of replica symmetry breaking and the putative spin glass phase of quantum gravity. ${ }^{2}$ However, it is reported in [11] that a naive application of the replica method leads to a pathological behavior of the free energy. In a recent paper [12] it is emphasized that the non-perturbative effect is important to resolve this problem.

In this paper, we will consider a simple toy model for the computation of free energy in JT gravity. Instead of the matrix model of JT gravity in [3], we consider the free energy in the Gaussian matrix model where the Hamiltonian is regarded as a random hermitian

\footnotetext{
${ }^{1}$ However, the recovering of a unitary Page curve is far from sufficient to completely resolve the paradox. We still lack an understanding of by what mechanism information is able to exit an evaporating black hole.

${ }^{2}$ The replica symmetry breaking in the SYK model is discussed in $[13,14]$. The idea of replica symmetry breaking is developed by Parisi $[15,16]$ to solve the sping glass model of Sherrington and Kirkpatrick [17]. See e.g. [18-20] for reviews of spin glasses.
} 
matrix with Gaussian distribution. ${ }^{3}$ We are interested in the so-called quenched free energy $\langle\log Z(\beta)\rangle,{ }^{4}$ in the matrix model, where $Z(\beta)=\operatorname{Tr} e^{-\beta H}$ is the partition function with the inverse temperature $\beta=T^{-1}$ and the expectation value is defined by the integral over the $N \times N$ hermitian matrix $H$

$$
\langle f(H)\rangle=\frac{\int d H e^{-\frac{N}{2} \operatorname{Tr} H^{2}} f(H)}{\int d H e^{-\frac{N}{2} \operatorname{Tr} H^{2}}} .
$$

One can compute the quenched free energy by the replica method ${ }^{5}$

$$
\langle\log Z(\beta)\rangle=\lim _{n \rightarrow 0} \frac{\left\langle Z(\beta)^{n}\right\rangle-1}{n} .
$$

In the high temperature regime the $n$-point correlator $\left\langle Z(\beta)^{n}\right\rangle$ is approximated by the disconnected correlator

$$
\left\langle Z(\beta)^{n}\right\rangle \approx\langle Z(\beta)\rangle^{n},
$$

and the $n \rightarrow 0$ limit in (1.2) gives rise to

$$
\langle\log Z(\beta)\rangle \approx \lim _{n \rightarrow 0} \frac{\langle Z(\beta)\rangle^{n}-1}{n}=\log \langle Z(\beta)\rangle .
$$

The right hand side of this equation is known as the annealed free energy. On the other hand, in the low temperature regime it is not clear how to define the analytic continuation of $\left\langle Z(\beta)^{n}\right\rangle$ to $n<1$. This is the origin of the difficulty found in [11].

It turns out that we can avoid this difficulty of analytic continuation by directly evaluating the quenched free energy by the matrix integral

$$
\langle\log Z(\beta)\rangle=\frac{\int d H e^{-\frac{N}{2} \operatorname{Tr} H^{2}} \log \operatorname{Tr} e^{-\beta H}}{\int d H e^{-\frac{N}{2} \operatorname{Tr} H^{2}}} .
$$

We can rewrite this integral (1.5) as an integral over the $N$ eigenvalues of the matrix $H$ and study the physical quantities like the free energy $F$ and the entropy $S$

$$
F=-T\langle\log Z(\beta)\rangle, \quad S=-\frac{\partial F}{\partial T} .
$$

In order for the entropy to be positive, the free energy $F$ should be a monotonically decreasing function of $T$. In the replica computation of the quenched free energy of JT gravity [11], a pathological non-monotonic behavior of $F$ is found under a certain prescription of the

\footnotetext{
${ }^{3}$ This problem is suggested in the discussion section in [11].

${ }^{4}$ Strictly speaking the quenched free energy is defined by including the factor of temperature $F=$ $-T\langle\log Z(\beta)\rangle$ as in (1.6), but we will loosely use the name "quenched free energy" to indicate either $\langle\log Z(\beta)\rangle$ or $F=-T\langle\log Z(\beta)\rangle$ depending on the context. We believe that which one we are referring to is clear from the context and this will not cause a confusion to the readers.

${ }^{5}$ The correlators of the resolvent $\operatorname{Tr}(E-H)^{-1}$ in the Gaussian matrix model are analyzed by the replica method in [21]. It is found that the replica symmetry breaking is important to reproduce the known results of the correlators of resolvents. In this paper we are dealing with the different quantity $\langle\log Z(\beta)\rangle$ and the computation in [21] cannot simply be generalized to our case.
} 
analytic continuation in $n .^{6}$ We find that the direct computation of the quenched free energy in the Gaussian matrix model (1.5) gives rise to a well-defined monotonic behavior of the free energy $F$.

This paper is organized as follows. In section 2, we find the explicit integral representation of the quenched free energy (1.5) and study its behavior in the high and low temperature regimes. In section 3 , we study the exact free energy and entropy for $N=2,3$ as examples. We find that the free energy exhibits a well-defined monotonic behavior as a function of $T$. In section 4 , we comment on the computation using the replica method. We propose a necessary condition for the analytic continuation of $\left\langle Z(\beta)^{n}\right\rangle$ to satisfy. Finally, we conclude in section 5 with some discussions on the interesting future problems.

\section{Quenched free energy in Gaussian matrix model}

In this paper we will analyze the quenched free energy in Gaussian matrix model (1.5) directly without using the replica trick. From the standard argument, the matrix integral in (1.5) is written as an integral over the $N$ eigenvalues $\left\{E_{1}, \cdots, E_{N}\right\}$ of $H$

$$
\langle\log Z(\beta)\rangle=\frac{1}{\mathcal{Z}} \frac{1}{N !} \int_{-\infty}^{\infty} \prod_{i=1}^{N} d E_{i} e^{-\frac{N}{2} E_{i}^{2}} \prod_{i<j}\left(E_{i}-E_{j}\right)^{2} \log \left(\sum_{i} e^{-\beta E_{i}}\right) .
$$

Here the normalization factor $\mathcal{Z}$ is given by

$$
\mathcal{Z}=\frac{1}{N !} \int_{-\infty}^{\infty} \prod_{i=1}^{N} d E_{i} e^{-\frac{N}{2} E_{i}^{2}} \prod_{i<j}\left(E_{i}-E_{j}\right)^{2}=N^{-\frac{N^{2}}{2}}(2 \pi)^{\frac{N}{2}} G_{2}(N+1),
$$

where $G_{2}(N+1)$ denotes the Barnes $G$-function. Using this expression (2.1), in subsection 2.1 and 2.2 we will study the behavior of quenched free energy in the high temperature and the low temperature regimes, respectively.

\subsection{High temperature regime}

In the high temperature regime, the quenched free energy is approximated by the annealed free energy (1.4).

The one-point function $\langle Z(\beta)\rangle$ in the Gaussian matrix model happens to be the same as the computation of the $1 / 2$ BPS Wilson loop in $\mathcal{N}=4$ super Yang-Mills theory (SYM), and the exact result at finite $N$ is found in [22] in terms of the Laguerre polynomial

$$
\langle Z(\beta)\rangle=e^{\frac{\beta^{2}}{2 N}} L_{N-1}^{1}\left(-\frac{\beta^{2}}{N}\right) .
$$

\footnotetext{
${ }^{6}$ As emphasized in [11] the analytic continuation of $\left\langle Z(\beta)^{n}\right\rangle$ from positive integer $n$ to $n<1$ is not unique. The non-monotonic behavior of the free energy is a consequence of the particular choice of the analytic continuation used in [11]. However, as explained in [11] their choice of analytic continuation is not meant to be the correct one but it is just an illustrative example to demonstrate the importance of the replica wormholes in the computation of free energy.
} 
The large $N$ behavior of the one-point function $\langle Z(\beta)\rangle$ can be computed from the genuszero eigenvalue density

$$
\rho_{0}(E)=\frac{1}{2 \pi} \sqrt{4-E^{2}},
$$

known as the Wigner semi-circle distribution. Then in the large $N$ limit the one-point function $\langle Z(\beta)\rangle$ becomes

$$
\langle Z(\beta)\rangle \approx N \int_{-2}^{2} d E \rho_{0}(E) e^{-\beta E}=N \frac{I_{1}(2 \beta)}{\beta},
$$

where $I_{1}(2 \beta)$ is the modified Bessel function of the first kind. From this expression one can easily find the expansion of the free energy and the entropy in the high temperature regime $(T \gg 1)$

$$
\begin{aligned}
& F=-T \log N-\frac{1}{2} T^{-1}+\mathcal{O}\left(T^{-3}\right), \\
& S=\log N-\frac{1}{2} T^{-2}+\mathcal{O}\left(T^{-4}\right) .
\end{aligned}
$$

In particular, the high temperature limit of entropy is $\log N$

$$
\lim _{T \rightarrow \infty} S=\log N
$$

This is expected since $N$ is the dimension of the Hilbert space and $\log N$ is the maximal entropy of the system.

\subsection{Low temperature regime}

Next let us consider the low temperature regime $(T \ll 1)$. In the low temperature limit $\beta \rightarrow \infty$, one can see that $\log \operatorname{Tr} e^{-\beta H}$ becomes

$$
\lim _{\beta \rightarrow \infty} \log \left(\sum_{i=1}^{N} e^{-\beta E_{i}}\right)=-\beta \min \left\{E_{i}\right\}
$$

where $\min \left\{E_{i}\right\}$ denotes the smallest eigenvalue in the set of $N$ eigenvalues $\left\{E_{1}, \cdots, E_{N}\right\}$. Thus we find that the low temperature limit of the quenched free energy is determined by the expectation value $E_{0}=\left\langle\min \left\{E_{i}\right\}\right\rangle$ of the smallest eigenvalue

$$
\lim _{\beta \rightarrow \infty}\langle\log Z(\beta)\rangle=-\beta E_{0} .
$$

Note that $E_{0}$ is explicitly written as the eigenvalue integral

$$
E_{0}=\frac{1}{\mathcal{Z}} \frac{1}{N !} \int_{-\infty}^{\infty} \prod_{i=1}^{N} d E_{i} e^{-\frac{N}{2} E_{i}^{2}} \prod_{i<j}\left(E_{i}-E_{j}\right)^{2} \min \left\{E_{i}\right\} .
$$

We do not know the closed form of this integral for general $N$, but it is possible to evaluate this integral for small $N$. For instance, for $N=2,3$ we find

$$
\begin{aligned}
& E_{0}^{(N=2)}=-\sqrt{\frac{2}{\pi}} \approx-0.79788, \\
& E_{0}^{(N=3)}=-\frac{9 \sqrt{3}}{8 \sqrt{\pi}} \approx-1.09936 .
\end{aligned}
$$


It is known [23] that in the large $N$ limit $E_{0}$ converges to the edge of the Wigner semi-circle distribution (2.4)

$$
E_{0}^{(N=\infty)}=-2 .
$$

It turns out that one can systematically compute the small $T$ corrections to the leading term in (2.9). In the eigenvalue integral (2.1), one can choose $E_{N}$ as the smallest eigenvalue without loss of generality. Then the range of other eigenvalues $E_{i}(i=1, \cdots, N-1)$ is restricted to $E_{i}>E_{N}$. With this remark in mind, the quenched free energy is written as

$$
\begin{aligned}
\langle\log Z(\beta)\rangle= & -\beta E_{0}+\frac{1}{\mathcal{Z}} \frac{N}{N !} \int_{-\infty}^{\infty} d E_{N} e^{-\frac{N}{2} E_{N}^{2}} \int_{E_{N}}^{\infty} \prod_{i=1}^{N-1} d E_{i} e^{-\frac{N}{2} E_{i}^{2}} \\
& \times \prod_{i=1}^{N-1}\left(E_{i}-E_{N}\right)^{2} \prod_{1 \leq i<j \leq N-1}\left(E_{i}-E_{j}\right)^{2} \log \left(1+\sum_{i=1}^{N-1} e^{-\beta\left(E_{i}-E_{N}\right)}\right) .
\end{aligned}
$$

This is further simplified by shifting $E_{i} \rightarrow E_{i}+E_{N}(i=1, \cdots, N-1)$ and integrating out $E_{N}$

$$
\begin{aligned}
\langle\log Z(\beta)\rangle= & -\beta E_{0}+\frac{1}{\mathcal{Z}} \frac{\sqrt{2 \pi}}{N !} \int_{0}^{\infty} \prod_{i=1}^{N-1} d E_{i} e^{-\frac{N}{2} \sum_{i} E_{i}^{2}+\frac{1}{2}\left(\sum_{i} E_{i}\right)^{2}} \\
& \times \prod_{i=1}^{N-1} E_{i}^{2} \prod_{1 \leq i<j \leq N-1}\left(E_{i}-E_{j}\right)^{2} \log \left(1+\sum_{i=1}^{N-1} e^{-\beta E_{i}}\right) .
\end{aligned}
$$

This is our master formula.

The small $T$ behavior of (2.14) is found by rescaling one of the integration variables $E_{i} \rightarrow T E_{i}$. In this way we find that the quenched free energy at low temperature $(T \ll 1)$ behaves as

$$
F=-T\langle\log Z(\beta)\rangle=E_{0}-\sigma T^{4}+\mathcal{O}\left(T^{5}\right),
$$

where $\sigma$ is an $N$-dependent constant. From (2.15), it follows that the entropy in the low temperature regime behaves as

$$
S=-\frac{\partial F}{\partial T}=4 \sigma T^{3}+\mathcal{O}\left(T^{4}\right) .
$$

This result implies that the entropy vanishes at zero temperature

$$
\lim _{T \rightarrow 0} S=0 .
$$

Note that the vanishing of the entropy at zero temperature is also observed in the Parisi's solution of Sherrington-Kirkpatrick model [24, 25].

\section{Numerics for $N=2,3$}

In this section we study numerically the integral representation of the quenched free energy (2.14) for $N=2,3$. For $N=2$ the integral (2.14) becomes

$$
\langle\log Z(\beta)\rangle=\sqrt{\frac{2}{\pi}} \beta+\sqrt{\frac{2}{\pi}} \int_{0}^{\infty} d E e^{-\frac{1}{2} E^{2}} E^{2} \log \left(1+e^{-\beta E}\right),
$$




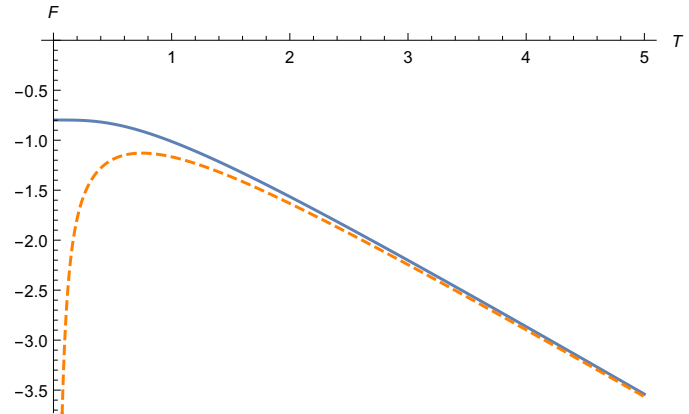

(a) Free energy at $N=2$

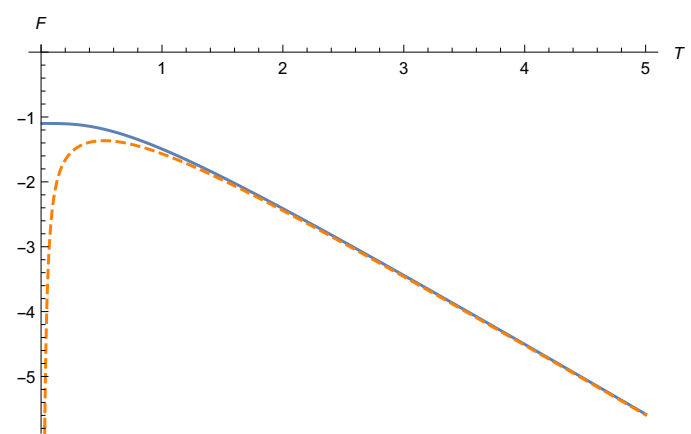

(b) Free energy at $N=3$

Figure 1. Plot of free energy for (a) $N=2$ and (b) $N=3$ as a function of temperature $T$. The solid curves are the quenched free energy while the orange dashed curves represent the annealed free energy $F_{\text {ann }}=-T \log \langle Z(\beta)\rangle$ with the exact one-point function in (2.3).

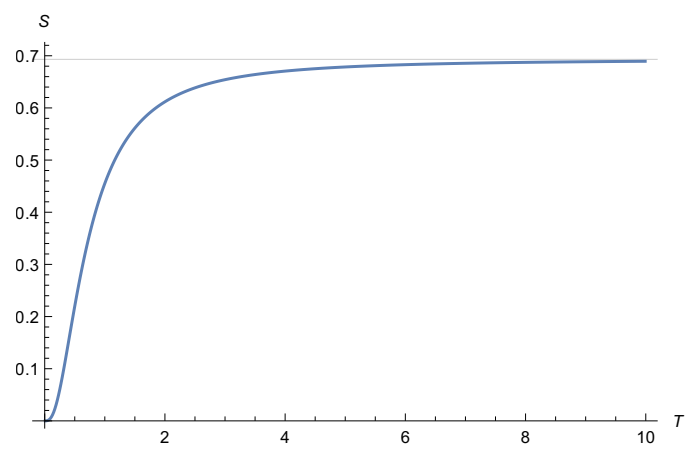

(a) Entropy at $N=2$

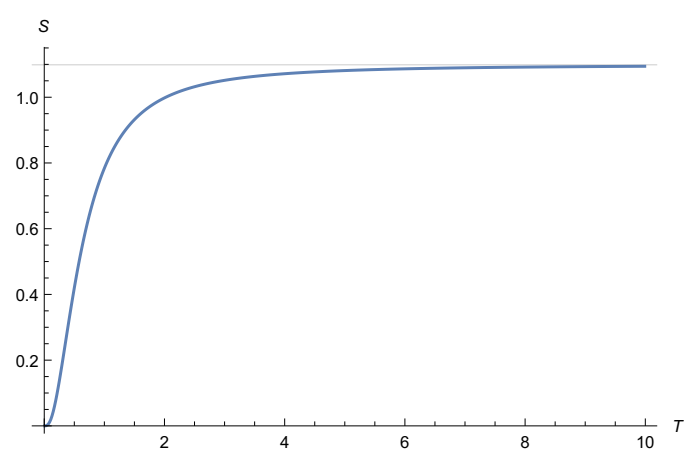

(b) Entropy at $N=3$

Figure 2. Plot of entropy for (a) $N=2$ and (b) $N=3$ as a function of temperature $T$. The solid curves are the exact result while the horizontal gray lines represent the maximum value of entropy $S=\log N$.

and for $N=3$ we find

$$
\begin{aligned}
& \langle\log Z(\beta)\rangle=\frac{9 \sqrt{3}}{8 \sqrt{\pi}} \beta \\
& +\frac{27 \sqrt{3}}{8 \pi} \int_{0}^{\infty} d E_{1} \int_{0}^{\infty} d E_{2} e^{-\frac{3}{2}\left(E_{1}^{2}+E_{2}^{2}\right)+\frac{1}{2}\left(E_{1}+E_{2}\right)^{2}} E_{1}^{2} E_{2}^{2}\left(E_{1}-E_{2}\right)^{2} \log \left(1+e^{-\beta E_{1}}+e^{-\beta E_{2}}\right) .
\end{aligned}
$$

One can easily evaluate these integrals numerically. In figure 1 we show the plot of free energy as a function of temperature. At high temperature, the quenched free energy approaches the annealed free energy $F_{\text {ann }}=-T \log \langle Z(\beta)\rangle$ (orange dashed curve) as expected. In figure 2 we show the plot of entropy $S$. One can see that $S$ approaches $\log N$ at high temperature and vanishes at zero temperature.

Let us take a closer look at the low temperature regime for $N=2$. The small $T$ expansion of the integral (3.1) is obtained by rescaling the integration variable $E \rightarrow T E$ 


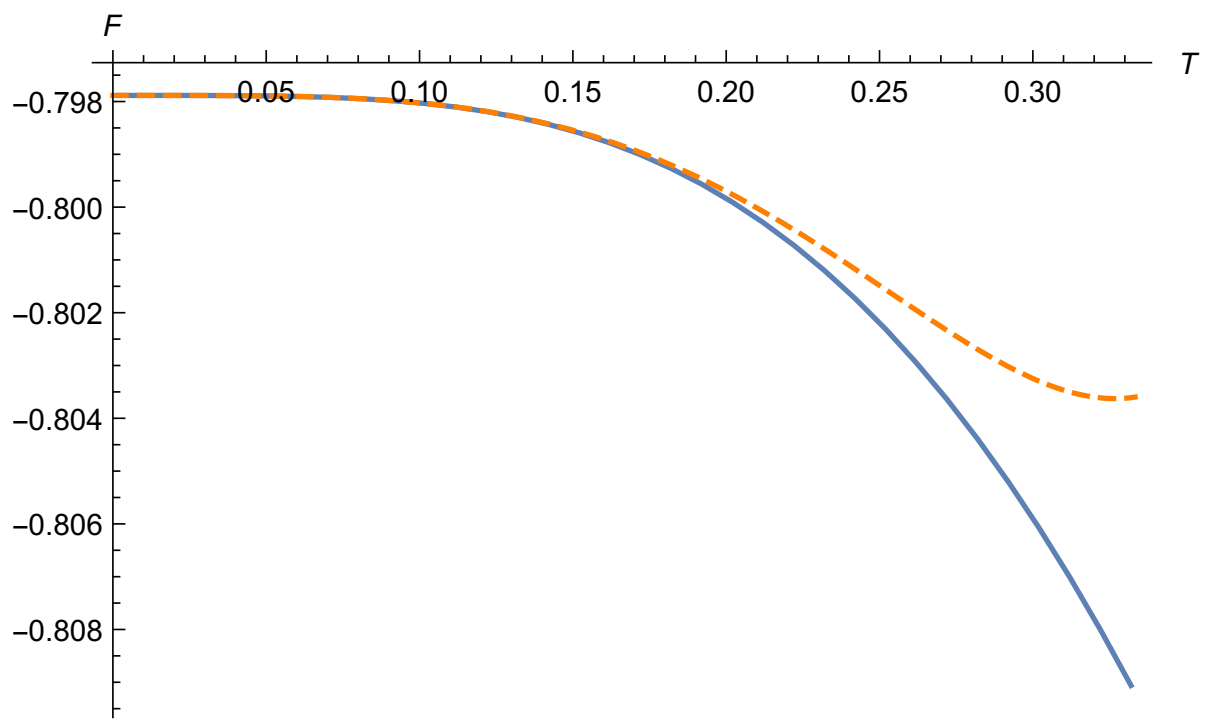

Figure 3. Plot of free energy for $N=2$ at low temperature. The solid curve is the exact result while the orange dashed curve represents the small $T$ expansion (3.3).

and expanding the Gaussian factor in (3.1)

$$
\begin{aligned}
F & =-\sqrt{\frac{2}{\pi}}\left[1+T^{4} \int_{0}^{\infty} d E E^{2} \sum_{n=0}^{\infty} \frac{1}{n !}\left(-\frac{T^{2} E^{2}}{2}\right)^{n} \log \left(1+e^{-E}\right)\right] \\
& =-\sqrt{\frac{2}{\pi}}\left[1+\frac{7 \pi^{4}}{360} T^{4}-\frac{31 \pi^{6}}{2520} T^{6}+\mathcal{O}\left(T^{8}\right)\right] .
\end{aligned}
$$

Note that the first small $T$ correction is of order $T^{4}$ which is consistent with the general result in (2.15). In figure 3 we show the plot of quenched free energy for $N=2$ and its small $T$ expansion up to $T^{6}$ in (3.3). One can see that the exact quenched free energy is a monotonic function of $T$ even in the low temperature regime and $F$ becomes $E_{0}$ at zero temperature. A pathological non-monotonic behavior found in [11] using the replica trick does not occur in the exact result of quenched free energy.

\section{Comment on the replica method}

Let us compare our direct calculation of quenched free energy with the replica method (1.2). As we mentioned in section 1, one can easily apply the replica method in the high temperature regime and obtain the result (1.4). In particular, in the high temperature limit $\beta \rightarrow 0$, the partition function $Z(\beta)=\operatorname{Tr} e^{-\beta H}$ reduces to the dimension of the Hilbert space

$$
\lim _{\beta \rightarrow 0} Z(\beta)=\operatorname{Tr} 1=N .
$$

Thus the quenched free energy approaches the maximal entropy of the system in the limit $T \rightarrow \infty$

$$
\lim _{\beta \rightarrow 0}\langle\log Z(\beta)\rangle=\lim _{n \rightarrow 0} \frac{N^{n}-1}{n}=\log N .
$$


On the other hand, the application of the replica trick in the low temperature regime is rather subtle. Under a certain prescription of the analytic continuation in the number of replicas $n$, it is found that the free energy exhibits a non-monotonic behavior as a function of temperature [11].

Our direct computation of the quenched free energy puts a certain constraint on the possible form of the analytic continuation in $n$. At low temperature, the smallest eigenvalue $E_{0}$ of $H$ becomes dominant and thus we expect

$$
\lim _{\beta \rightarrow \infty}\left\langle Z(\beta)^{n}\right\rangle=e^{-n \beta E_{0}} .
$$

We can regard (4.3) as a condition for the possible analytic continuation of $\left\langle Z(\beta)^{n}\right\rangle$ to satisfy. Then we can apply the replica method in the low temperature regime

$$
\lim _{\beta \rightarrow \infty}\langle\log Z(\beta)\rangle=\lim _{n \rightarrow 0} \frac{e^{-n \beta E_{0}}-1}{n}=-\beta E_{0},
$$

which reproduces the correct behavior of the quenched free energy (2.9).

Note that there is no $\log N$ entropy term in (4.4) since only a single eigenvalue (the lowest energy state) contributes to $\left\langle Z(\beta)^{n}\right\rangle$ in the low temperature limit. This explains the vanishing of entropy at zero temperature (2.17).

We would like to understand the role of replica symmetry breaking in a possible large $N$ phase transition. When $n$ is a positive integer, the $n$-replica correlator $\left\langle Z(\beta)^{n}\right\rangle$ is expanded in terms of the connected correlators

$$
\left\langle Z(\beta)^{n}\right\rangle=n ! \sum_{\sum p \nu_{p}=n} \prod_{p=1}^{n} \frac{1}{\nu_{p} !(p !)^{\nu_{p}}}\left(\left\langle Z(\beta)^{p}\right\rangle_{\mathrm{conn}}\right)^{\nu_{p}} .
$$

Here $\left[p^{\nu_{p}}\right]=\left[1^{\nu_{1}} 2^{\nu_{2}} \cdots n^{\nu_{n}}\right]$ denotes a partition of $n$. In the high temperature regime the disconnected part $\langle Z(\beta)\rangle^{n}$ corresponding to the partition $\left[1^{n}\right]$ is dominant, while at low temperature the totally connected part $\left\langle Z(\beta)^{n}\right\rangle_{\text {conn }}$ corresponding to the partition $\left[n^{1}\right]$ is dominant [26]. Then one might naively think that the quenched free energy in the low temperature regime is given by the totally connected correlator $\left\langle Z(\beta)^{n}\right\rangle_{\text {conn }}$

$$
\langle\log Z(\beta)\rangle=\lim _{n \rightarrow 0} \frac{\left\langle Z(\beta)^{n}\right\rangle_{\mathrm{conn}}-1}{n} .
$$

One can try to compute $\left\langle Z(\beta)^{n}\right\rangle_{\text {conn }}$ for integer $n$ and analytically continue it to $n=0$. However, this analytic continuation is very subtle since $\left\langle Z(\beta)^{n}\right\rangle_{\text {conn }}$ scales as $N^{2-n}$ in the large $N$ limit and the naive $n \rightarrow 0$ limit of $\left\langle Z(\beta)^{n}\right\rangle_{\text {conn }}$ is not 1 and the limit (4.6) does not exist. It is not clear how to define the analytic continuation of $\left\langle Z(\beta)^{n}\right\rangle_{\text {conn }}$ which satisfies the condition (4.3). We believe that (4.6) is not the correct way to compute the low temperature regime of quenched free energy. In other words, the two limits $\beta \rightarrow \infty$ and $n \rightarrow 0$ do not commute.

A similar problem has appeared in the so-called random energy model $[27] .{ }^{7}$ In [27] this problem is circumvented by promoting $\left(p, \nu_{p}\right)$ in $(4.5)$ as a continuous variable and the

\footnotetext{
${ }^{7}$ The random energy model is defined as a model with $N$ randomly distributed energy eigenvalues with Gaussian distribution but the correlation among eigenvalues is ignored. It is known that the random energy model is equivalent to the $p \rightarrow \infty$ limit of a $p$-spin generalization of the Sherrington-Kirkpatrick model [27, 28].
} 
correct low temperature behavior is obtained by plugging $\nu_{p}=\frac{n}{p}$ and extremizing the term $\left(\left\langle Z(\beta)^{p}\right\rangle_{\text {conn }}\right)^{n / p}$ in (4.5) with respect to $p$. The $n$-point function $\left\langle Z(\beta)^{n}\right\rangle$ obtained with this prescription indeed satisfies the necessary condition (4.3) and we can safely take the $n \rightarrow 0$ limit [27]. The resulting quenched free energy $F$ exhibits a phase transition in the large $N$ limit at a certain critical temperature $T_{c}$ : for $T>T_{c}, F$ agrees with the annealed free energy which takes the form $F_{\text {ann }}=a T+b T^{-1}$ with some coefficients $a, b$, while for $T<T_{c}, F$ is constant [27]. This $F$ is a monotonic function of $T$ as expected. It would be interesting to see if the same prescription works in the present case of random matrix model. We leave this as an interesting future problem.

\section{Discussion}

In this paper we have analyzed the quenched free energy in Gaussian matrix model directly without using the replica method. We find an integral representation of the exact quenched free energy (2.14). The exact quenched free energy is a monotonic function of temperature as expected, and the entropy computed from this free energy approaches $\log N$ at high temperature and vanishes at zero temperature.

There are many interesting open questions. It is very interesting to see if there is a phase transition in the large $N$ limit. In the case of random energy model, it is known that there is a phase transition associated with the replica symmetry breaking and the low temperature phase corresponds to a spin glass [29]. Since the random matrix model considered in this paper can be thought of as a generalization of the random energy model, it is tempting to speculate that the quenched free energy of the random matrix model also exhibits a phase transition. ${ }^{8}$ To settle this issue it is important to understand the analytic continuation of $\left\langle Z(\beta)^{n}\right\rangle$ to $n<1$. We proposed a simple condition (4.3) for the analytic continuation of $\left\langle Z(\beta)^{n}\right\rangle$ to satisfy.

It would be very interesting to generalize our analysis to the JT gravity matrix model and see if the spin glass phase is realized at low temperature [11]. In [11] the quenched free energy is computed by a certain prescription of the analytic continuation of $\left\langle Z(\beta)^{n}\right\rangle$ and it leads to a pathological behavior at low temperature. It is argued in [12] that this problem is resolved by including the non-perturbative effect. It would be very interesting to complete the program of replica computation of the free energy in JT gravity.

Our analysis suggests that at low temperature the smallest eigenvalue (or the lowest energy state) gives a dominant contribution to the quenched free energy. This reminds us of the "eigenbrane" introduced in [30]. Perhaps the spacetime picture of the low temperature phase is described by an eigenbrane with one of the eigenvalues pinned to the edge of the spectral density. It would be interesting to investigate this picture further.

\footnotetext{
${ }^{8}$ As discussed in [26], all contributions in the decomposition (4.5) become comparable around $T \sim N^{-2 / 3}$ and the dominance of disconnected part $\langle Z(\beta)\rangle^{n}$ is lost below this temperature. In the strict $N \rightarrow \infty$ limit this temperature $T \sim N^{-2 / 3}$ vanishes. To keep this scale finite, we can take a scaling limit $N \rightarrow \infty, T \rightarrow 0$ with $T N^{2 / 3}$ fixed. As discussed in [11], this amounts to focusing on the edge of the Wigner distribution (2.4) and this scaling limit corresponds to the so-called Airy limit.
} 


\section{Acknowledgments}

This work was supported in part by JSPS KAKENHI Grant No. 19K03845.

Open Access. This article is distributed under the terms of the Creative Commons Attribution License (CC-BY 4.0), which permits any use, distribution and reproduction in any medium, provided the original author(s) and source are credited.

\section{References}

[1] R. Jackiw, Lower Dimensional Gravity, Nucl. Phys. B 252 (1985) 343 [InSPIRE].

[2] C. Teitelboim, Gravitation and Hamiltonian Structure in Two Space-Time Dimensions, Phys. Lett. B 126 (1983) 41 [INSPIRE].

[3] P. Saad, S.H. Shenker and D. Stanford, JT gravity as a matrix integral, arXiv:1903.11115 [INSPIRE].

[4] P. Saad, S.H. Shenker and D. Stanford, A semiclassical ramp in SYK and in gravity, arXiv: 1806.06840 [INSPIRE].

[5] A.M. García-García and J.J.M. Verbaarschot, Spectral and thermodynamic properties of the Sachdev-Ye-Kitaev model, Phys. Rev. D 94 (2016) 126010 [arXiv:1610.03816] [InSPIRE].

[6] J.S. Cotler et al., Black Holes and Random Matrices, JHEP 05 (2017) 118 [Erratum ibid. 09 (2018) 002] [arXiv: 1611.04650] [INSPIRE].

[7] S. Sachdev and J. Ye, Gapless spin fluid ground state in a random, quantum Heisenberg magnet, Phys. Rev. Lett. 70 (1993) 3339 [cond-mat/9212030] [INSPIRE].

[8] A. Kitaev, A simple model of quantum holography (part 1 and 2), talks at Kavli Institute for Theoretical Physics, Santa Barbara U.S.A., on 7 April 2015:

http://online.kitp.ucsb.edu/online/entangled15/kitaev/ and on 27 May 2015: http://online.kitp.ucsb.edu/online/entangled15/kitaev2/.

[9] G. Penington, S.H. Shenker, D. Stanford and Z. Yang, Replica wormholes and the black hole interior, arXiv: 1911.11977 [INSPIRE].

[10] A. Almheiri, T. Hartman, J. Maldacena, E. Shaghoulian and A. Tajdini, Replica Wormholes and the Entropy of Hawking Radiation, JHEP 05 (2020) 013 [arXiv:1911.12333] [INSPIRE].

[11] N. Engelhardt, S. Fischetti and A. Maloney, Free Energy from Replica Wormholes, arXiv: 2007.07444 [INSPIRE].

[12] C.V. Johnson, Low Energy Thermodynamics of JT Gravity and Supergravity, arXiv: 2008.13120 [INSPIRE].

[13] G. Gur-Ari, R. Mahajan and A. Vaezi, Does the SYK model have a spin glass phase?, JHEP 11 (2018) 070 [arXiv: 1806.10145] [INSPIRE].

[14] I. Aref'eva, M. Khramtsov, M. Tikhanovskaya and I. Volovich, Replica-nondiagonal solutions in the SYK model, JHEP 07 (2019) 113 [arXiv:1811.04831] [INSPIRE].

[15] G. Parisi, An Infinite Number of Order Parameters for Spin Glasses, Phys. Rev. Lett. 43 (1979) 1754 [INSPIRE].

[16] G. Parisi, A sequence of approximated solutions to the SK model for spin glasses, J. Phys. A 13 (1980) L115. 
[17] D. Sherrington and S. Kirkpatrick, Solvable Model of a Spin-Glass, Phys. Rev. Lett. 35 (1975) 1792 [INSPIRE].

[18] F. Denef, TASI lectures on complex structures, in Theoretical Advanced Study Institute in Elementary Particle Physics: String theory and its Applications: From meV to the Planck Scale, World Scientific, Singapore (2011), pg. 407 [arXiv: 1104.0254] [INSPIRE].

[19] T. Castellani and A. Cavagna, Spin-glass theory for pedestrians, J. Stat. Mech. 2005 (2005) P05012 [cond-mat/0505032].

[20] D. Sherrington, Spin Glasses, cond-mat/9806289.

[21] A. Kamenev and M. Mézard, Wigner-Dyson statistics from the replica method, J. Phys. A 32 (1999) 4373 [cond-mat/9901110].

[22] N. Drukker and D.J. Gross, An Exact prediction of $N=4$ SUSYM theory for string theory, J. Math. Phys. 42 (2001) 2896 [hep-th/0010274] [INSPIRE].

[23] Z.-D. Bai and Y.-Q. Yin, Necessary and sufficient conditions for almost sure convergence of the largest eigenvalue of a Wigner matrix, Ann. Probab. (1988) 1729.

[24] A. Crisanti and T. Rizzo, Analysis of the $\infty$-replica symmetry breaking solution of the Sherrington-Kirkpatrick model, Phys. Rev. E 65 (2002) 046137.

[25] H.-J. Sommers and W. Dupont, Distribution of frozen fields in the mean-field theory of spin glasses, J. Phys. C 17 (1984) 5785.

[26] K. Okuyama, Replica symmetry breaking in random matrix model: a toy model of wormhole networks, Phys. Lett. B 803 (2020) 135280 [arXiv:1903.11776] [INSPIRE].

[27] B. Derrida, Random-energy model: An exactly solvable model of disordered systems, Phys. Rev. B 24 (1981) 2613 [inSPIRE].

[28] B. Derrida, Random-Energy Model: Limit of a Family of Disordered Models, Phys. Rev. Lett. 45 (1980) 79 [INSPIRE].

[29] D.J. Gross and M. Mezard, The Simplest Spin Glass, Nucl. Phys. B 240 (1984) 431 [INSPIRE].

[30] A. Blommaert, T.G. Mertens and H. Verschelde, Eigenbranes in Jackiw-Teitelboim gravity, arXiv:1911.11603 [INSPIRE]. 